\title{
Association of the altered sense of time in the seconds-to-minute range with intertemporal choices across time-horizons
}

\author{
Evgeniya Lukinova ${ }^{1,2}$ and Jeffrey C. Erlich ${ }^{1,2,3}$ \\ ${ }^{1}$ NYU-ECNU Institute of Brain and Cognitive Science at NYU Shanghai, China \\ ${ }^{2}$ NYU Shanghai, Shanghai, China \\ ${ }^{3}$ Shanghai Key Laboratory of Brain Functional Genomics (Ministry of Education), East China Normal University, Shanghai, \\ China
}

\begin{abstract}
Delay-discounting studies in neuroscience, psychology, and economics have been mostly focused on concepts of self-control, reward evaluation, and discounting. Another important relationship to consider is the link between intertemporal choice and time perception. We presented 50 college students with timing exercises on the range of seconds to minutes and intertemporalchoice tasks on both the time-scale of seconds and of days. We hypothesized that individual differences in time perception would influence decisions about short experienced delays but not long delays. While we found some evidence that individual differences in internal clock speed accounts for some unexplained variance between choices across time-horizons, overall our findings suggest a nominal contribution of the altered sense of time in intertemporal choice.
\end{abstract}

Keywords: intertemporal choice, time perception, time production, internal clock speed

How humans and animals internally represent time is an active research topic (Paton \& Buonomano, 2018). Calendar time differs from its internal chronological perception (Ray \& Bossaerts, 2011), called "biological time". In fact, because human time perception varies unpredictably from the calendar time, external observers who base their judgment on objective time, naturally call some people 'impulsive' while others 'patient' (Lucci, 2013). Supporting this intuitive link between sense of time and impulsivity, a handful of studies suggest that individual differences in timing modulate delay discounting behavior. Of those studies, some have suggested

Evgeniya Lukinova https://orcid.org/0000-0002-8357-9307

Jeffrey C. Erlich https://orcid.org/0000-0001-9073-7986

We acknowledge two outstanding undergraduates who helped us to start this project: Huixin Yan from UCSD and Danielle John from CUNY Hunter College. We thank NYU Shanghai undergraduate students Muzi Du, Xue Bai, and Weiyi He who helped us to collect the data. Funding was provided by Shanghai Eastern Scholar Program (Shanghai Municipal Education Commission) to E.L. J.C.E. acknowledges the support of the NYU-ECNU Institute of Brain and Cognitive Science at NYU Shanghai and the 111 Project, Base B16018. This study has been preregistered at OSF (https://osf.io/vaqf8). Software for running the task, as well as the de-identified data and analysis code for regenerating our results are available on GitHub (https://www.github.com/erlichlab/delayTP).

Correspondence concerning this article should be addressed to Jeffrey C. Erlich, NYU Shanghai, 1555 Century Avenue, Pudong New District, Shanghai, China 200122. Contact: jerlich@nyu.edu that nonlinearities in biological time explain why discounting appears hyperbolic rather than exponential (Bradford, Dolan, \& Galizzi, 2019; Kim \& Zauberman, 2009; Takahashi, Oono, \& Radford, 2008). Others have related individual timing to intertemporal choice tasks behavior, but use both hypothetical timing (i.e. "How long is 1 month?") and hypothetical delay discounting tasks (Zauberman, Kim, Malkoc, \& Bettman, 2009). To date, we know only one study (Corvi, Juergensen, Weaver, \& Demaree, 2012) that has explored timing and intertemporal choice (both not hypothetical) in the seconds-to-minutes range.

In a recent study, we compared subjects' delay discounting choices for offers in seconds and days (Lukinova, Wang, Lehrer, \& Erlich, 2019). We found that the choices in the days task explained around $40 \%$ of the variance of choices in the seconds task: a substantial portion, but also leaving the majority of variance unexplained. Given that a substantial ( $60 \%$ ) fraction of the variance in one task was not explained by the other task, we considered differences in the tasks that could contribute to the unshared variance. One important difference was that all delays in the seconds task were experienced during the session (e.g., if a subject chose 10 coins in 30 seconds, then she would have to sit and wait for 30 seconds to receive the coins before proceeding to the next trial). In contrast, in the days task each choice was recorded and at the end of the session one trial was implemented. If the subject chose a delayed reward on that trial, they went about their lives and received an electronic payment at the appropriate time. Thus, individual differences in timing seem more likely to influence choices in the seconds task than in 
the days task.

Here, we used both the intertemporal choice task and measured time perception and production. Our withinsubject experiment directly addresses the question, posed by Wittmann and Paulus (2008), whether variation in timing in the seconds-to-minutes range influences not only discounting in seconds, but also discounting in days, weeks, and months. In our experiment, we compare timing on the range of seconds-to-minutes with delay discounting on the timescale of seconds and of days, importantly, both not hypothetical: in the timing tasks subjects had to report perceived time and produce intervals and in the discounting task subjects' payment depended on the choices they made.

In the seconds-to-minutes range the dominant model of temporal processing has been the internal clock model (Church, 1984; Karmarkar \& Buonomano, 2007). This model suggests that the pulse count provides a linear metric of time and following temporal judgments rely on comparing the current pulse count to that of a reference time. Timing not only of longer intervals but also of intervals lasting from one second to tens of seconds appears consistent with mechanisms that generate a linear metric of time (Karmarkar \& Buonomano, 2007). Nevertheless, there is no consensus in the literature whether subjects with higher or lower (than average) internal clock speed (ICS) are more impulsive. People with higher (lower) ICS perceive time passing faster (slower) than a stopwatch (e.g., for an objective 30 seconds period one might subjectively report $35 \mathrm{~s}$ (25s) elapsing). Some scholars suggest that impulsive decisions might be due to the fact that time intervals are perceived subjectively as lasting too long (higher ICS: Baumann \& Odum, 2012; van den Broek, Bradshaw, \& Szabadi, 1992; Wittmann \& Paulus, 2008). Others suggest that a higher internal clock speed might mean an increased cognitive processing speed that "allows for a more deliberate processing of the choice presented" (Sharma \& Khan, 2018) and, thus, subjects favor waiting (Corvi et al., 2012). Moreover, when forming intertemporal preferences on the scales larger than minutes, subjective estimates of duration might not accurately map onto objective time (Zauberman et al., 2009). Nevertheless, there seems to be some consensus that it is not higher ICS, but rather less accurate ICS that is correlated with impulsivity (Corvi et al., 2012).

We have four main hypotheses (preregistered at https://osf.io/vaqf8). First, that the ICS accounts for some of the variance in delay discounting, but only for delays that are experienced, i.e. on the scale of seconds. Second, subjects who appear more impulsive in the seconds task than the days task (measured via the difference in discount factors in $1 / \mathrm{sec}$ and in $1 /$ day, respectively) will have fast internal clocks and those who appear less impulsive in seconds than days will have slow internal clocks. Third, if subjects' delay discounting choices are fit with a model that ignores inter-subject variability in ICS, then subjects' ICS will contribute to the variance in measured discount factors. Fourth, if the subjects' delay discounting choices are fit with a model that accounts for subjective differences in internal clock, the correlation between second and day discount rates will increase.

We found that timing had additive value in explaining the time-horizons gap in discounting, but only when the time perception estimates were done at the same time as the decision-making choices and when the time perception task proxy for ICS was used. However, we did not significantly improve correlation between discounting on the time-scale of seconds and of days after accounting for subjective time. Together, these results suggest that variation in ICS can contribute to small variations in estimated discount rates, but the degree of contributed variation is small enough that it seems reasonable to ignore under most circumstances.

\section{Method}

This study has been preregistered at OSF (https://osf.io/vaqf8).

\section{Participants}

As preregistered, we invited two groups of subjects: a 'follow-up' group (subjects that participated previously in the main or control experiment 1 of Lukinova et al. (2019) and a new (named 'naive' in the preregistration) group of subjects (Figure 1A-B). We decided to recruit new subjects for two reasons. First, we anticipated the challenge of bringing back the desired number of subjects. Second, in case of variation over more than 1 year of time preferences or timing properties, we might not be able to test whether timing is related to discounting task (since the latter took place more than a year before the data collection for this study).

For the new group, 30 participants (as preregistered) were recruited from the NYU Shanghai undergraduate student population. Four subjects were excluded from all analyses because their choices were insensitive to delay leading to a total sample of 26 (16 women, 10 men) NYU Shanghai students. The study was approved by the IRB of NYU Shanghai. The subjects were between 18-21 years old, 13 subjects were Chinese nationals. Subjects received a $40 \mathrm{CNY}(\sim \$ 5.5$ USD) per hour participation fee as well as up to an additional $50 \mathrm{CNY}$ ( $\sim 8 \mathrm{USD})$ per session based on their individual performance in the tasks. The experiment involved two sessions per subject, permitting us to perform withinsubject analyses. The sessions were scheduled bi-weekly and took place in the NYU Shanghai Behavioral and Experimental Economics Laboratory in Shanghai. In each session, all decisions in the intertemporal choice task involved a choice between a later (delay in seconds and days) option and an immediate (now) option. During the second session the experiment included the intertemporal choice task and the timing tasks, in this order. The follow-up group only did not redo 
the discounting task. We compared their previous choices in discounting (Lukinova et al., 2019) with newly collected data from the timing task.

Corvi et al. (2012) reported $r=.43, p<.01$ between discount factor and ICS error, and $r=-.31$ between discount factor and ICS. Thus, we expected an $r$ value from .3 to .5 for the correlation between timing variables and discount factor in seconds. A power analysis indicates that for expected correlation $r=.5$ and $80 \%$ power (the ability of a test to detect an effect, if the effect actually exists) the required sample size is $N=29$, for a medium size correlation of $r=.3$ the required sample size is $N=84$ (Cohen, 2013). We preregistered collection and analysis of data from 30 participants in each experimental group. However, we were able to bring back only 24 participants for follow-up group (19 women, 5 men; between 20-24 years old; 14 subjects were Chinese Nationals). Therefore, a total of 50 (out of 54) subjects with 30 (5 time intervals $\times 3$ repetitions $x 2$ tasks) timing observations per each subject was considered for analysis. This is a within-subject study, so all conditions applied equally to all subjects.

\section{Materials and Procedure}

In our timing experiment, we used two tasks to measure each subject's timing properties (Figure 1D-E). They were always in order: first, a time perception task, then, a time production task. We used this order since the time intervals for estimation were the same as those for production and we did not want to bias our subjects with any numbers prior to assessing their time perception. All subjects first participated in a demonstration with instructions and two trials of each task (interval $=10$ seconds) to facilitate the real experiment.

For the time perception task the instruction was: "Welcome to the experiment. You will estimate how long the circle has shown up and type in your estimation as an integer (unit $=$ seconds). Please press [space] key to start the experiment when you are ready." The time intervals that were used in the task are 3, 7, 14, 30, and 64 seconds, each repeated 3 times, in random order.

For the time production task the instruction was: "Welcome to the experiment. You will press the [enter] key to start and press the [enter] key to end the estimation of the given time intervals. Please press [space] key to start the experiment when you are ready." The time intervals that were used in the task are $3,7,14,30$, and 64 seconds, each repeated 3 times, in random order.

In both timing tasks we did not provide instructions 'not to count' (Rattat \& Droit-Volet, 2012). In earlier pilot studies, subjects reported that this instruction was hard to follow and resulted in unreliable data. Both tasks took approximately 12-15 minutes to finish. The duration of each task (not including the real timing distortions and inter-trial intervals that depend on each subject's speed) was $(3+7+14+30+64)$ x 3/60 = 5.9 minutes. Although some experimenters point to a repetition effect affecting the time estimates, according to Matthews (2011), effects disappear when there is a modest lag between presentations (in our case, five different stimuli in random order). Also, Mioni, Stablum, McClintock, and Grondin (2014) revealed that time production techniques are not equivalent, with the method involving key presses to start and stop the production (which we use) showing the highest accuracy.

The intertemporal task for new participants mimicked the last two sessions in Lukinova et al. (2019) control experiment 1 'no circles' (Figure 1C). Two sessions (two weeks apart) included an alternating set of verbal tasks (verbal short delay, SV, and verbal long delay, LV): SV-LV-SV-LV (or LVSV-LV-SV, for a random half of subjects). The payment was done differently for SV and LV: in the former, subjects accumulated coins and the total earned was paid via electronic payment at the end of each experimental session, in the latter, a single trial was selected at random at the conclusion of the session for payment. In our sessions, the exchange rate in $\mathrm{SV}$ was $0.05 \mathrm{CNY}$ per coin (since all coins are accumulated and subjects are paid the total profit), whereas in LV, the exchange rate was $4 \mathrm{CNY}$ per coin. These exchange rates were set to, on average, equalize the possible total profit between short and long delays tasks. Across sessions, 200 trials were conducted in verbal short delay (SV, 3-64 seconds) and verbal long delay (LV, 3-64 days).

\section{Analysis}

Preregistered Analysis. Timing experiments resulted in several observations per subject in the time perception and time production tasks. Some researchers used only a production task and calculated the ICS as the ratio of produced versus actual duration (Glicksohn \& Hadad, 2012). Since we used two tasks we preregistered to convert observations from both tasks into our key variables following the procedure from Corvi et al. (2012).

First, per each time interval, the average time estimation ( $T e$ ) and average time production $(T p)$ were calculated by averaging three trials for the same time interval. Then, the ratios TeRatio and TpRatio were calculated by dividing $T e$ and $T p$ by the actual time interval duration, respectively as suggested by Glicksohn and Hadad (2012). Next, we adjusted the procedure, since Corvi et al. (2012) used only one interval per task, and averaged the ratios across time intervals in each task (MTeRatio and MT pRatio).

In general, people with higher than average internal clock speed (ICS) estimate an objective duration to be longer than average and tend to produce shorter durations than average (Church, 1984). We decided to separately consider data from the production task and the perception task as proxies for ICS. According to the definition, the calculation of the proxy 
from time perception (estimation) task was straight-forward:

$$
\text { ICSe = MTeRatio }
$$

Thus, ICS values higher and lower than 1 indicate internal clock speeds faster and slower than objective time, respectively. For the production task we need to symmetrically reflect the value of the averaged timing ratio around 1 in order to adhere to the same meaning of the ICS. So for $M T$ pRatio $>1$ the proxy is calculated as ICS $p=$ $(1-a b s(M T$ pRatio -1$))$ and MT pRatio $<1$ the proxy is calculated as ICS $p=(1+a b s(M T p R a t i o-1))$ (the latter definition was preregistered). Combined, this gives us the equation below for the time production proxy of ICS:

$$
\text { ICS } p=2-M T \text { pRatio }
$$

The ICS error was calculated as:

$$
\text { ICS Error }=(a b s(M T e \text { Ratio }-1)+a b s(M T \text { pRatio }-1)) / 2
$$

thus, higher values indicate greater error.

We called the variables ICS e, ICS p, and ICS Error, as defined above, the 'timing variables'. For plotting the distributions of the timing variables (Figure 3D-F) we calculated probability density estimates (for smoothing) using the ksdensity function in Matlab. By default the estimate is based on a normal kernel function, and is evaluated at equally-spaced 100 points, $x_{i}$, that cover the range of the data in $x$.

In the preregistration, we did not expect subjects' time production to be distorted more than two times. However, one of our subjects produced an interval of more than 160 seconds when asked to produce 64 seconds. In this case, Equation 2 cannot convert the MT pRatio to a proxy of ICS correctly. This subject did adhere to the scalar properties and was not removed from the overall analysis. However, whenever proxies for ICS and ICS Err were used, this subject's data were removed.

In order to test our preregistered hypotheses, we compared timing variables to the discount factors in short and long tasks. The discount factors for the new subjects were estimated using a softmax-hyperbolic fit in a similar way as in Lukinova et al. (2019), i.e. a four population level and three subject level parameters model (mixed-effects model) is used with the help of 'brms' package in R (Bürkner, 2017) that allows to do BHM of nonlinear multilevel models in Stan with the standard R formula syntax (Carpenter et al., 2016; Guo et al., 2016):

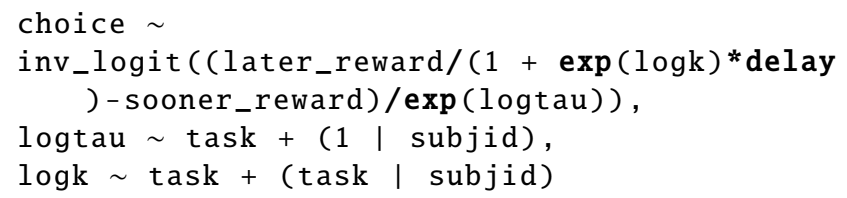

where later_reward is the later reward, sooner_reward is the sooner reward; logk is the natural logarithm of the discounting parameter $k$ and $\log$ tau $(\log (\tau))$ is the $\log$ of the decision noise. Fitting $e^{\log (k)}$ allows $\log (k)$ to vary from $-\infty$ to $+\infty$ while $k=e^{\log (k)}$ is restricted to $[0,+\infty]$. The model $\left(M_{4 p, 3 s}\right)$ had 4 population level parameters $(\log (k)$ and $\log (\tau)$ for each of the two intertemporal choice tasks) and 3 parameters per subject: $\log \left(k_{S V}\right), \log \left(k_{L V}\right)$ and $\log (\tau)$. R package shinystan (Gabry, 2015) was used to diagnose and develop the models.

Following our general hypotheses, we preregistered to specifically examine whether there are:

1. individual differences in short verbal discount factor, but not long verbal discount factor, that are accounted for by differences in timing variables;

2. a positive correlation between ICS Error and discount factor (bigger error $\sim$ more impulsive) for short delays;

3. a higher correlation between discount factors in short and long delays after accounting for subjective timing in the short delay task.

We used the following linear models to test the contribution of timing variables to each discount factor:

$$
\begin{aligned}
& \log \left(K_{S V}\right) \sim I C S e+I C S p+I C S \text { Error }+\log \left(K_{L V}\right) \\
& \log \left(K_{L V}\right) \sim I C S e+I C S p+I C S \text { Error }+\log \left(K_{S V}\right)
\end{aligned}
$$

This regression analysis addressed the first two hypotheses. $\mathrm{R}$ package lme4 was used for linear models (Bates, Maechler, Bolker, \& Walker, 2014).

To convert objective time into subjective time we considered both power and linear forms of subjective timing. The functional form for the power law was

$$
S T(t)=\alpha \cdot t^{\beta}
$$

where $S T$ is the subjective time, $t$ is the target (actual) duration, $\alpha$ is a linear scaling in producing (or estimating) durations, and $\beta$ captures the degree of nonlinearity (Glicksohn \& Hadad, 2012; Kim \& Zauberman, 2009). We also planned the estimation of a special case of the power function, where $\beta=1$.

$$
S T(t)=\alpha \cdot t
$$

Then $\alpha$, the slope of a linear function, reflected the change in the produced (or in the estimated) duration for a unit change in the target duration (considered as another index of ICS by Sharma \& Khan, 2018; Wittmann \& Paulus, 2008). The higher the slope, the more time is produced (or less estimated) for a unit change in the target duration reflecting a slower internal clock.

Other planned analyses are reported in the Supplemental Online Material and included: (a) testing the scalar properties of timing; (b) performing nonparametric tests to compare 
timing variables between genders and correlations of timing variables with the Barratt Impulsiveness Scale (BIS).

The permutation tests of differences between the means of two groups are done by shuffling the group label and computing the mean between the shuffled groups 10000 times. This generates a null distribution which is used to estimate the probability of observing the true difference between groups (bootmean in https://github.com/erlichlab/elutils).

Additional Analysis. In order to do a kfold comparison between the models and test the difference in correlation between the discount factors in objective and subjective time for the follow-up group we refit the subsample from Lukinova et al. (2019) following the same procedure. We use the refit values only for Table 6 and Supplemental Online Material. Therefore, note that the correlation coefficient for the subsample from Lukinova et al. (2019) (Pearson $r=.49$, $p=.014$ ) is different (but not significantly different according to 'cocor' tests) from the one reported in the refitted objective time model (Pearson $r=.43, p=.034)$. Additionally, we fit the BHM for all participants (the follow-up and the new groups combined). The discount factors align well across the fits (see Supplemental Online Material).

\section{Software}

The code for the timing experiment was written in Python using the 'PsychoPy' toolbox (version 1.83.04; Peirce, 2007). The code for the intertemporal choice task was re-used from Lukinova et al. (2019) available at https://www.github.com/erlichlab/delay3ways. All analyses and statistics were performed either in Matlab (version 9.3, or higher, The Mathworks, MA), or in R (version 3.4.1, or higher, R Foundation for Statistical Computing, Vienna, Austria). R package brms (2.0.1) was used as a wrapper for Rstan to perform Bayesian nonlinear multilevel modeling. $\mathrm{R}$ package stargazer (Hlavac, 2018) is used to transform $\mathrm{R}$ regression results to LaTex tables.

\section{Results}

The subjects in this study were 50 undergraduate students from NYU Shanghai. One group, the "follow-up" group ( $N=24)$, consisted of subjects from Lukinova et al. (2019) who originally participated in five delay discounting experimental sessions and then did a sixth session (timing experiment) one half to two years later (Figure 1A and Supplemental Online Material). The second "new" group $(N=26)$ participated in two experimental sessions (Figure 1B), with two weeks in between the sessions. The sessions under consideration in this paper either included verbal intertemporal choice task, the timing experiment, or both.

In each trial of the intertemporal choice task, subjects made a decision between a sooner and a later option (Figure $1 \mathrm{C}$; as in Lukinova et al., 2019). For the short delay tasks, when subjects chose the later option, a clock appeared on the screen, and only when the clock image disappeared, could they obtain their reward, visualized as a stack of coins. The visual presentation of coins was accompanied by a 'dropping coins' sound. At the end of the session, all coins were converted to local currency (CNY) as payment to the subject. In the long delay task, after each choice subjects were given feedback confirming their choice, but only one trial was selected randomly and converted to CNY to determine the subject's payment.

In each trial of the timing experiment, subjects typed in their estimation of the duration of a circle that was shown on the screen (time perception task, also called 'time estimation', Figure 1D) or produced a time interval by pressing a key twice (to start and to end the production) on the keyboard reflecting a given time interval (time production task, Figure 1E). Subjects received a fixed payment (40 CNY) for the timing experiment.

\section{Subjects' time preferences are reliable across second/day differences}

Subjects' time preferences were estimated by fitting their choices with a Bayesian hierarchical model (BHM) of hyperbolic discounting with decision noise (as in Lukinova et al., 2019). The model had four population level parameters: $\log$ discount factor, $\log (k)$, and the $\log$ of the decision noise, $\log (\tau)$ for both intertemporal choice tasks; and three parameters per subject: $\log \left(k_{S V}\right), \log \left(k_{L V}\right)$ and $\log (\tau)$. We used this model to fit 10269 choices across 26 subjects in the new group. Subjects' choices were well-fit by the model (Figure $2 \mathrm{C}$ for an example subject and Supplemental Online Material for all subjects).

Within the new group, we found a high correlation between short delay verbal task (SV) and long delay verbal task (LV; Figure 2A; Pearson $r=.63, p<.001$ ). This replicated our earlier finding (see Figure 3B in Lukinova et al., 2019). The follow-up group is a subsample from Lukinova et al. (2019). This subgroup also demonstrated a strong correlation between choices in SV and LV (Pearson $r=.49, p=.014$ ).

\section{Subjects' timing varies monotonically with the actual time intervals}

Subjects' internal timing properties were examined in several ways. The average time estimation $(T e)$ and average time production $(T p)$ were calculated by averaging three trials for the same time interval (see three example subjects in Figure 3A-C and all subjects in Supplemental Online Material). Two proxies for ICS were calculated using ICS $e$ and $I C S p$ measures (e.g., for ICS $e$, first dividing $T e$ by the actual time interval duration and then averaging across different time intervals, Equation 1, and for ICS $p$ we used Equation 2 to reflect the value (calculated after the same procedure as for ICS e) symmetrically around 1, Method). The ICS error was calculated by capturing distortions from the unity line, 
A Follow-up from Lukinova et al. 2019

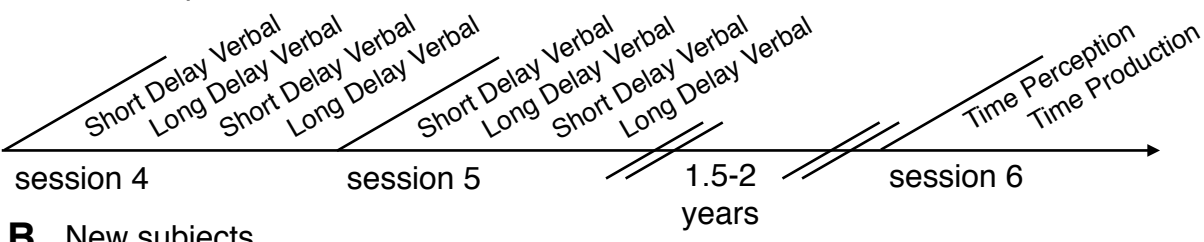

B New subjects
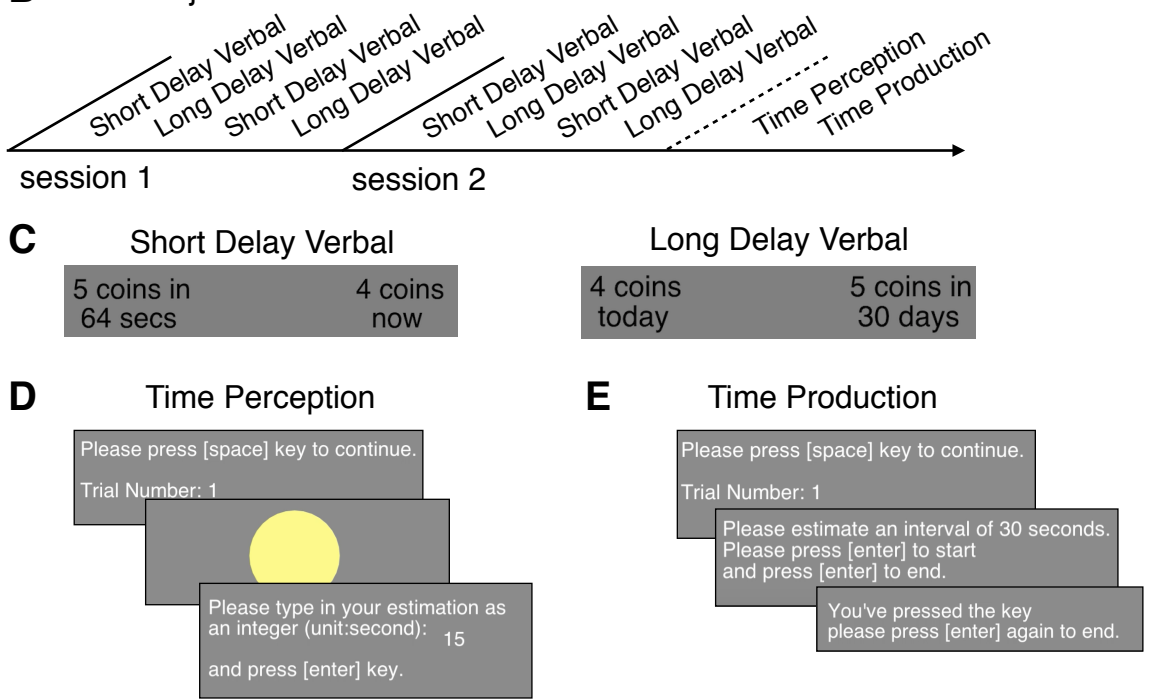

Figure 1. Behavioral Tasks. (A) Timeline of experimental sessions for the follow-up group. (B) Timeline of experimental sessions for the new group. (C) Stimuli examples in the intertemporal choice task. (D-E) Screenshots of the timing experiment.

where $\mathrm{x}$-axis is Actual Time and $\mathrm{y}$-axis is Reported Time (Equation 3). We fit each subjects' estimation and production (separately) with power and linear functions (Equation 6 and Equation 7, respectively) of the actual time.

Almost all subjects reported times varied monotonically with the actual time interval (Figure 3A-C and Supplemental Online Material). We also observed an increase in variance with increased time intervals, as expected from scalar timing. We arranged subjects into three categories by their ICS: lower ICS (Figure 3A), higher ICS (Figure 3C) and almost perfect ICS (compared to objective time, defined as $0.98<$ ICSe < 1.02, e.g., in (Figure 3B; Table 1). The majority of our subjects had a lower ICS, meaning that they would perceive time passing slower than a stopwatch (e.g., for an objective 30 seconds period she might subjectively report 25 seconds elapsing). Surprisingly, one subject in the follow-up group produced intervals that were more than twice the actual interval: e.g. produced a 160 secs interval for an actual interval of 64 secs. Therefore, for this person the definitions of ICS $p$ and the ICS Err (Equation 2 and Equation 3, respectively) are not correct. Thus, we decided to exclude this subject only from analyses that involve ICS proxies and ICS error measurement.

All subjects' subjective timing was fit well (using a BHM, Supplemental Online Material) with both linear and power functions. The exponent of the power function (Eq. 6), $\beta$,
Table 1

Internal clock speed

\begin{tabular}{lrrrrr}
\hline & \multicolumn{2}{c}{ ICS } & & \multicolumn{2}{c}{ ICS $p$} \\
\cline { 2 - 3 } \cline { 5 - 6 } & Follow-up & New & & Follow-up & New \\
\hline Lower ICS & 16 & 19 & & 14 & 16 \\
Higher ICS & 5 & 5 & & 7 & 5 \\
Perfect & 2 & 2 & & 2 & 5 \\
\hline Total & 23 & 26 & & 23 & 26 \\
\hline
\end{tabular}

is close to 1 for many subjects so the power and linear fits overlap (Figure 3A-C and Supplemental Online Material). According to 10-fold cross validation criteria ('kfold' model comparison in Supplemental Online Material) time estimation was better fit with a power function and time production with a linear function. Therefore, for models of subjective timing further on we use both linear and power fits.

\section{Timing explains variation in discounting only when mea- sured proximally}

Having estimated subjects' time preferences and timing properties we tested for correlations between them. We found no significant relationships between ICSe, ICS $p$, ICS Error and discount factor $(N=49$, Figure 4). Further- 
A

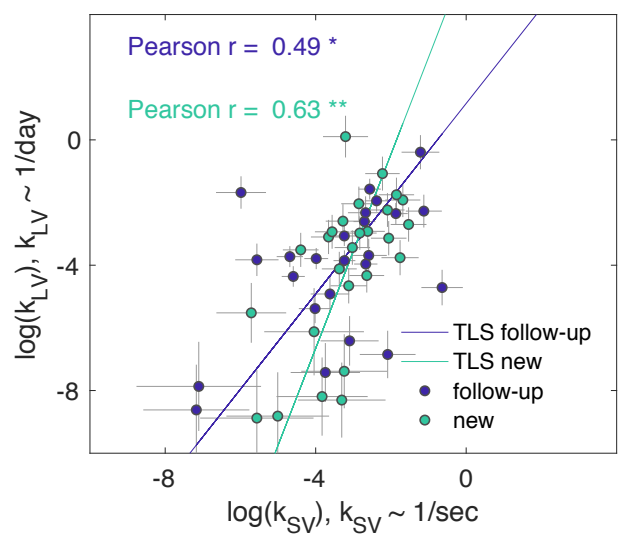

B

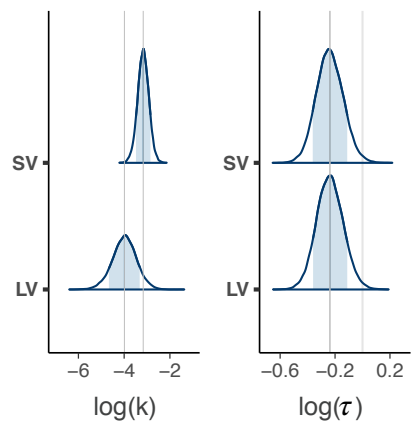

\section{C}
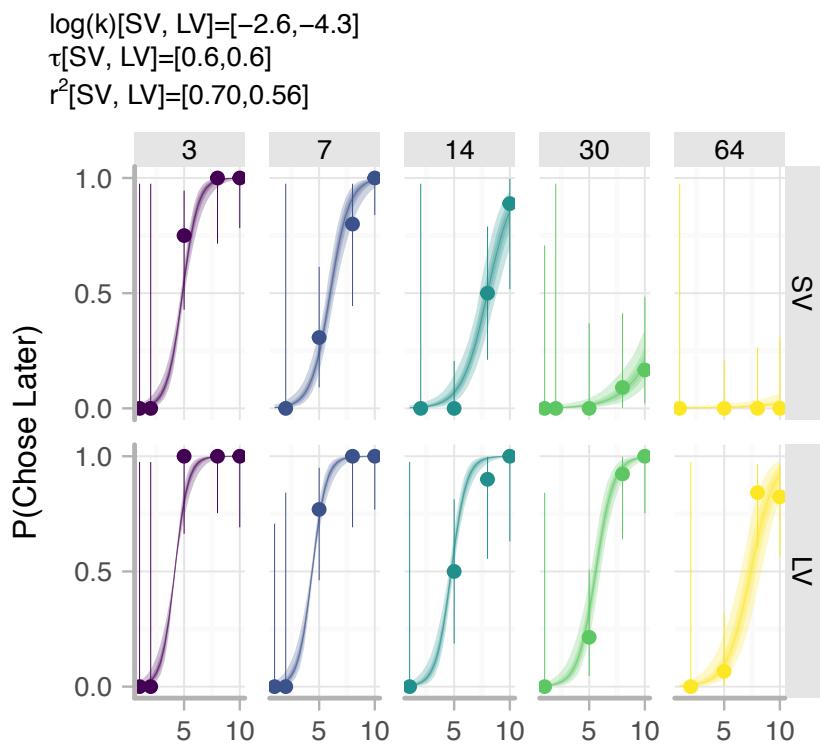

Reward Magnitude

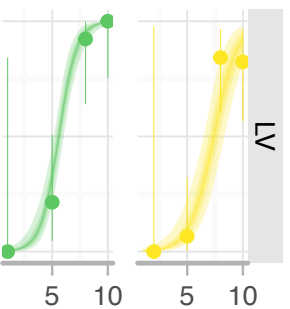

Figure 2. Comparison of discount factors across groups and tasks. (A) Each circle is one subject $(N=50)$. The logs of discount factors in short delay verbal task (SV, x-axis) plotted against the logs of discount factors in long delay verbal task (LV, y-axis). Discount factors are estimated in the units of the task. The color of the circles identify the group, either followup (purple) or new (cyan). The error bars are the $S D$ of the estimated coefficients (posterior means). The lines represent the perpendicular (or total) least squares (TLS) regression lines for two groups of subjects in its color, respectively (Huang, Rattner, Liu, \& Nathans, 2013). (B) Distribution of posterior parameter estimates of $\log (k)$ and $\log (\tau)$ from the model fit for the two tasks and two groups of subjects. (C) An example softmax-hyperbolic fit of one subject. In each panel, the marker and error bar indicate the mean and binomial confidence intervals of the subject's choices for that offer. The smooth ribbon indicated the BHM model fits (at 50, 80, 99\% credible intervals). At the top of the subject plot we indicate the mean estimates of $\log (k)$ and $\tau$ for each task for that subject. We also indicate the Bayesian $R^{2}$ for each task.

more, when we separated subjects who appear more impulsive in the seconds task than the days task and vice versa, we did not find that the groups are significantly different from each other using ICSe as proxy for ICS (Figure 4C, F; permutation test between $K_{L V}>K_{S V}$ and $K_{S V}>K_{L V}$ subgroups for the follow-up group, $M_{K_{L V}>K_{S V}}=0.87$ and $M_{K_{S V}>K_{L V}}=0.84$, gives $p=.624$ and for the new group,
$M_{K_{L V}>K_{S V}}=0.83$ and $\left.M_{K_{S V}>K_{L V}}=0.94, p=.178\right)$.

In our previous work (Lukinova et al., 2019) we established that $\log \left(k_{S V}\right)$ significantly predicted $\log \left(k_{L V}\right)$ and vice versa. Here, we preregistered a regression analysis to check whether timing might account for variance in discount factor from one task beyond what was explained by the discount factor in the other task. 
A

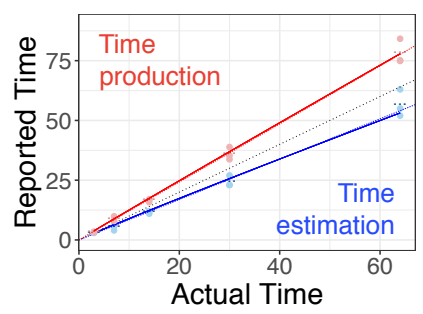

D

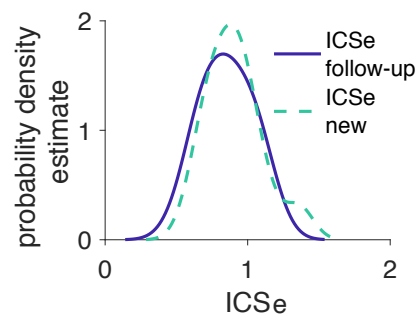

B

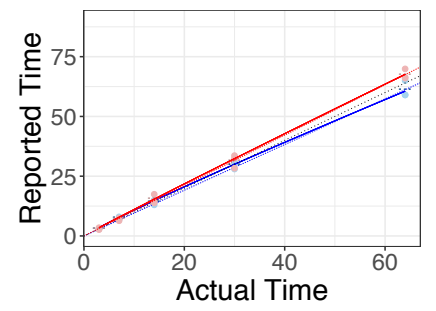

$\mathbf{E}$

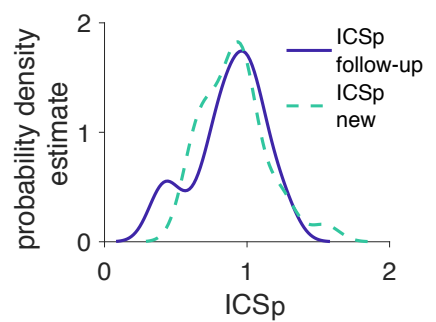

C

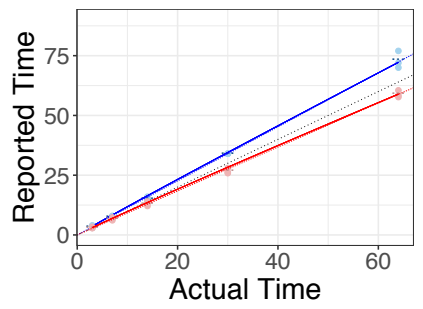

$\mathbf{F}$

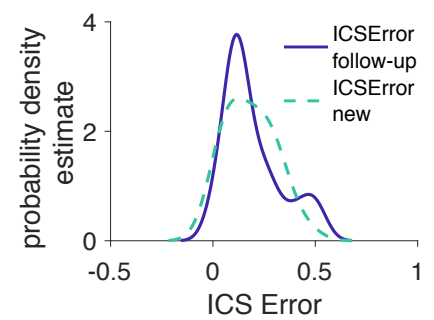

Figure 3. (A-C) Reported vs. actual time for three example subjects in the timing tasks. The color indicates the two different timing tasks: time perception (estimation) in blue and time production in red. Circles in each plot correspond to individual trials: three estimations and three productions per actual time interval. The horizontal dash is the average per time interval: $T e$ in dark blue and $T p$ in dark red for estimation and production, respectively. The dotted gray line is the unity line $y=x$. The solid and dashed lines of each color are the power and the linear fits, respectively. If the perceived time is slower (blue below $y=x$ ) and the produced time is faster (red above $y=x$ ) than a stopwatch that is consistent evidence for a lower ICS. (A) An example subject with a lower ICS. (B) An example subject with almost perfect ICS. (C) An example subject with higher ICS. (D-F) Kernel density estimation of the three timing variables $(N=49)$ with follow-up group in solid purple and new group in dashed cyan. The follow-up and the new group are not significantly different according to permutation tests for each of the three timing variables. (D) Distribution of ICS $e$ estimated using the time perception task as a proxy for ICS (follow-up $M=0.85$ vs. new $M=0.90$, permutation test, $p=.346$ ); (E) Distribution of ICS $p$ estimated using the time production task as proxy for ICS (follow-up $M=0.88$ vs. new $M=0.92$, permutation test, $p=.595$ ); (F) ICS error (follow-up $M=0.19$ vs. new $M=0.18$, permutation test, $p=.770)$.

To this end, we ran linear regressions according to Equation 4 and Equation 5. We tested the contribution of each factor by dropping it from the model to create a reduced nested model and performing a likelihood ratio test against the full model $(N=49$, Figure 5). We found some evidence that subjects' timing in the new group, but not in the follow-up group, was related to their discount factors. Dropping ICSe (a proxy for ICS) resulted in a significant decrease in the likelihood for explaining short delay ( $N=26$, Figure 5C), but not long delay task in the new group. Thus, we found some support for our hypothesis that ICS accounts for some of the variance in delay discounting for short experienced delays. With the addition of ICS $e$ we explained $48 \%$ (an increase in $8 \%$ compared to $40 \%$ for reduced model) of the variance in $K_{S}$ (Table 2). Detailed results of the regression analyses are presented in Tables 2-5.

\section{Accounting for subjective timing does not improve fits nor does it change the correlation between discount fac- tors significantly}

If individual differences in ICS influenced choices in the short delay discounting task, then using subjective time, rather than objective time should improve our ability to predict subjects' choices. To this end, we compared the model with objective delays ('obj') to four models with subjective delays:

- 'subjTep' - where delays in seconds were substituted by the power fits based on time estimation,

- 'subjTel' - where delays in seconds were substituted by the linear fits based on time estimation,

- 'subjTpp' - where delays in seconds were substituted by the power fits based on time production, and

- 'subjTel' - where delays in seconds were substituted by the linear fits based on time production. 


\section{Follow-up}

A

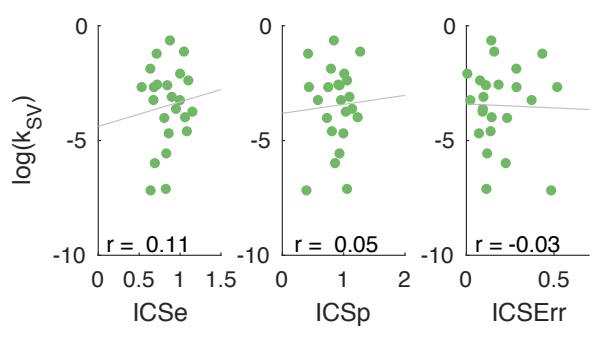

\section{New}

D

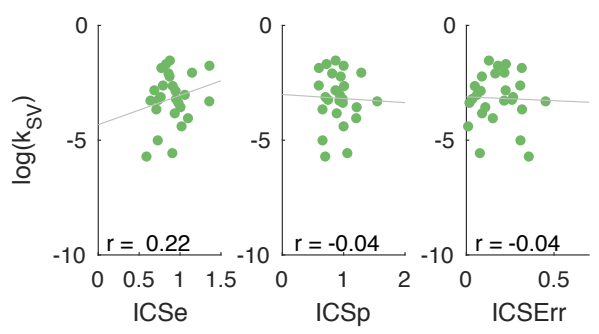

B

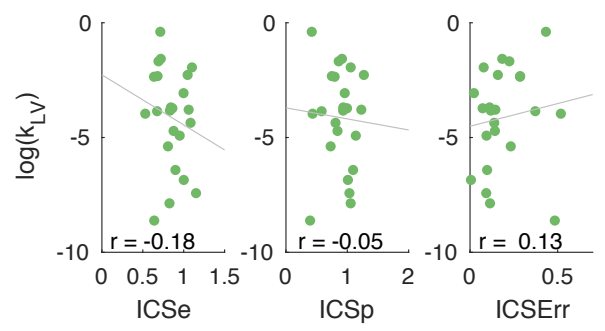

E

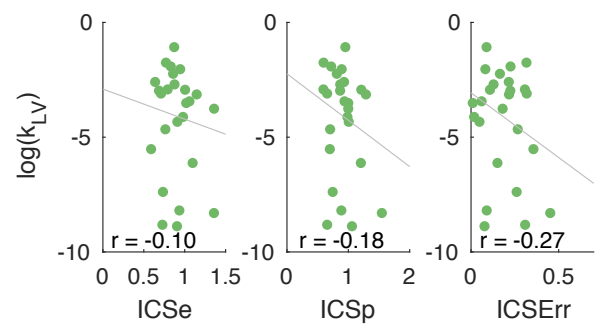

C

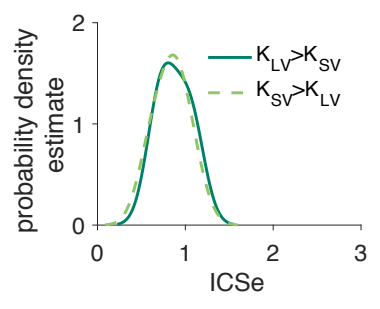

F

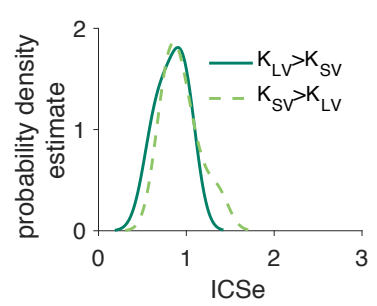

Figure 4. (A-B, D-E) Correlations between discount factors (y-axis) with ICS e, ICS p, and ICS Err (x-axis). Each circle is one subject ( $N=23$ - follow-up, $N=26$ - new group). Pearson's $r$ is reported on the figure (all $p>.05$ ). Best linear fit line $(y \sim x)$ is displayed. (C, F) Kernel density estimation of ICS $e$ as proxy for ICS for two subgroups of subjects divided by positive or negative difference between discount factor in short and long delay tasks.

A

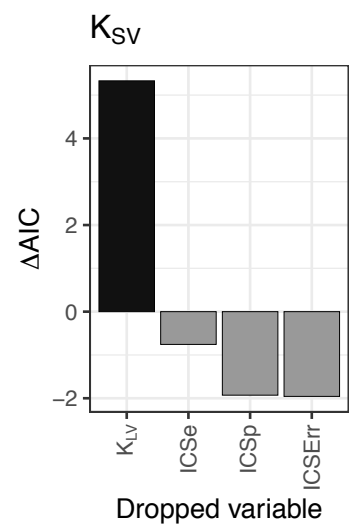

B

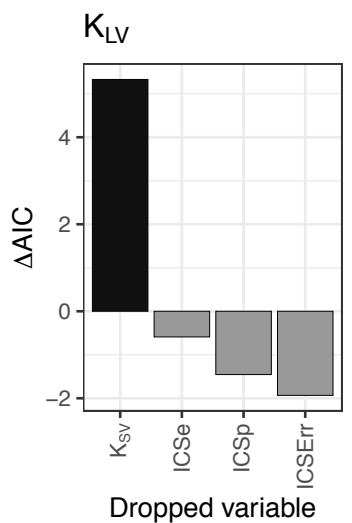

C

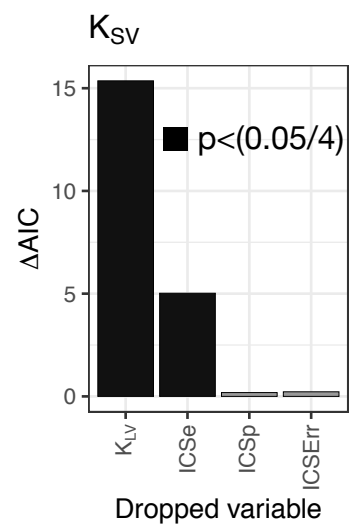

D

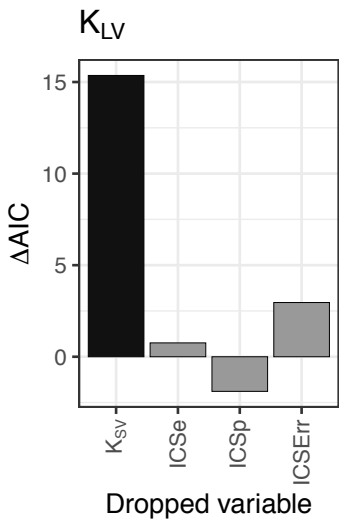

Figure 5. Drop-one regression analysis. We generated linear regression models of $\log (k)$ for each task (verbal short delay and verbal long delay) against the discount factor of the other task, as well as timing variables. In order to test which factors were important, we dropped each factor and tested whether the decrease in likelihood was significant by a $\chi^{2}$ test. (Analyses were done in R using the 'drop1' function). We plot the change in AIC, with significant drops in black $(p<.0125$, Bonferroni Corrected $p<.05 / 4)$. (A, B) - follow-up group; (C, D) - new group. 
Table 2

New Group $\log \left(k_{S V}\right)$ Regression Results

\begin{tabular}{|c|c|c|c|}
\hline & \multicolumn{3}{|c|}{ Dependent variable: $\log \left(k_{S V}\right)$} \\
\hline & (1) & (2) & (3) \\
\hline $\log \left(k_{L V}\right)$ & $\begin{array}{c}0.313^{* *} \\
(0.070)\end{array}$ & $\begin{array}{c}0.286^{* *} \\
(0.072)\end{array}$ & $\begin{array}{c}0.300^{* *} \\
(0.069)\end{array}$ \\
\hline ICSe & $\begin{array}{c}3.364^{*} \\
(1.318)\end{array}$ & & $\begin{array}{c}1.671 \\
(0.875)\end{array}$ \\
\hline ICSp & $\begin{array}{c}-1.559 \\
(1.149)\end{array}$ & & \\
\hline ICSErr & $\begin{array}{c}2.019 \\
(1.475)\end{array}$ & & \\
\hline Constant & $\begin{array}{c}-3.872^{* *} \\
(0.888)\end{array}$ & $\begin{array}{c}-2.001^{* *} \\
(0.342)\end{array}$ & $\begin{array}{c}-3.457^{* *} \\
(0.829)\end{array}$ \\
\hline Observations & 26 & 26 & 26 \\
\hline $\mathrm{R}^{2}$ & .567 & .397 & .480 \\
\hline Adjusted $\mathrm{R}^{2}$ & .484 & .372 & .435 \\
\hline Residual Std. Error & $0.806(\mathrm{df}=21)$ & $0.889(\mathrm{df}=24)$ & $0.844(\mathrm{df}=23)$ \\
\hline F Statistic & $6.865^{* *}(\mathrm{df}=4 ; 21)$ & $15.822^{* *}(\mathrm{df}=1 ; 24)$ & $10.607^{* *}(\mathrm{df}=2 ; 23)$ \\
\hline
\end{tabular}

Table 3

Follow-up Group $\log \left(k_{S V}\right)$ Regression Results

\begin{tabular}{lcc}
\hline \hline & \multicolumn{2}{c}{ Dependent variable: $\log \left(k_{S V}\right)$} \\
\cline { 2 - 3 } & $(1)$ & $(2)$ \\
\hline $\log \left(k_{L V}\right)$ & $0.433^{*}$ & $0.398^{*}$ \\
& $(0.166)$ & $(0.155)$ \\
ICSe & 3.359 & \\
& $(3.359)$ & \\
ICSp & -0.690 & \\
& $(2.922)$ & \\
ICSErr & 0.977 & $(0.720)$ \\
& $(5.291)$ & 23 \\
Constant & -4.132 & 0.239 \\
& $(4.315)$ & 0.203 \\
Observations & 23 & $1.575(\mathrm{df}=21)$ \\
$\mathrm{R}^{2}$ & 0.287 & $6.587^{* *}(\mathrm{df}=1 ; 21)$ \\
Adjusted $\mathrm{R}^{2}$ & 0.129 & ${ }^{*} \mathrm{p}<.05 ;{ }^{* *} \mathrm{p}<.01$ \\
Res. Std. Err. & $1.646(\mathrm{df}=18)$ & \\
F Statistic & $1.811(\mathrm{df}=4 ; 18)$ & \\
\hline Note: & & \\
& &
\end{tabular}

After accounting for subjective timing in the short delay task we did find a higher (but not significantly higher) correlation between discount factors in the short and long delay tasks (Table 6). This is only true in the models that use time perception (estimation) task. The significant differ-
Table 4

New Group $\log \left(k_{L V}\right)$ Regression Results

\begin{tabular}{lcc}
\hline \hline & \multicolumn{2}{c}{ Dependent variable: $\log \left(k_{L V}\right)$} \\
\cline { 2 - 3 } & $(1)$ & $(2)$ \\
\hline $\log \left(k_{S V}\right)$ & $1.555^{* *}$ & $1.388^{* *}$ \\
ICSe & $(0.348)$ & $(0.349)$ \\
& -4.888 & \\
ICSp & $(3.189)$ & \\
& 0.792 & \\
ICSErr & $(2.665)$ & 0.313 \\
& $-6.551^{*}$ & $(1.171)$ \\
Constant & $(3.118)$ & 26 \\
& $5.725^{*}$ & .397 \\
Observations & $(2.429)$ & .372 \\
$\mathrm{R}^{2}$ & 26 & $1.958(\mathrm{df}=24)$ \\
Adjusted $\mathrm{R}^{2}$ & .556 & $15.822^{* *}(\mathrm{df}=1 ; 24)$ \\
Residual Std. Error & .471 & ${ }^{*} \mathrm{p}<.05 ;{ }^{* *} \mathrm{p}<.01$ \\
F Statistic & $1.797(\mathrm{df}=21)$ & \\
\hline Note: & $6.574^{* *}(\mathrm{df}=4 ; 21)$ & \\
& & \\
& &
\end{tabular}

ence in correlations (between the objective model and four subjective time models) was tested using R package 'cocor' (Diedenhofen \& Musch, 2015) assuming nonoverlapping dependent correlations. 
Table 5

Follow-up Group $\log \left(k_{L V}\right)$ Regression Results

\begin{tabular}{lcc}
\hline & \multicolumn{2}{c}{ Dependent variable: $\log \left(k_{L V}\right)$} \\
\cline { 2 - 3 } & $(1)$ & $(2)$ \\
\hline $\log \left(k_{S V}\right)$ & $0.631^{*}$ & $0.600^{*}$ \\
& $(0.243)$ & $(0.234)$ \\
ICSe & -4.311 & \\
& $(4.042)$ & \\
ICSp & 2.294 & \\
& $(3.492)$ & $-2.047^{*}$ \\
ICSErr & 1.458 & $(0.906)$ \\
& $(6.386)$ & 23 \\
Constant & -0.557 & 0.239 \\
& $(5.339)$ & 0.203 \\
Observations & 23 & $1.933(\mathrm{df}=21)$ \\
$\mathrm{R}^{2}$ & 0.310 & \\
Adjusted $\mathrm{R}^{2}$ & 0.157 & ${ }^{*} \mathrm{p}<.05 ;{ }^{* *} \mathrm{p}<.01$ \\
Res. Std. Error & $1.988(\mathrm{df}=18)$ & \\
F Statistic & $2.023(\mathrm{df}=4 ; 18)$ & $6.587^{* *}(\mathrm{df}=1 ; 21)$ \\
\hline Note: & &
\end{tabular}

Table 6

Pearson Correlation between $\log \left(k_{S V}\right)$ and $\log \left(k_{L V}\right)$ Pearson $r \quad p \quad$ Sig. Better?

\begin{tabular}{lrrl}
\hline new & & & \\
obj & .63 & $<.001$ & \\
subjTel & .64 & $<.001$ & no \\
subjTep & .64 & $<.001$ & no \\
subjTpl & .51 & .008 & no \\
subjTpp & .51 & .007 & no \\
\hline follow-up & & & \\
obj & .43 & .034 & no \\
subjTel & .48 & .016 & no \\
subjTep & .48 & .018 & no \\
subjTpl & .39 & .063 & no \\
subjTpp & .41 & .046 & answers whether there is significant in- \\
Note. 'Sig. Better?' & answe to the re- \\
crease in correlations from the objective time model to the &
\end{tabular}

\section{Discussion}

Scholars have argued that it is crucial to combine timing and intertemporal choice research (Lucci, 2013; Rung, Frye, DeHart, \& Odum, 2019; Schultz, 2010; Wittmann \& Paulus, 2008). In this paper, we have shown that accounting for subjects' timing helps to predict discounting, but only using variation in time perception to explain variance in short delay tasks and when timing and discounting are proximally assessed. In the new group of subjects, accounting for timing explained an additional $8 \%$ of variance in discount factors in the seconds task: an effect which supported one of our main hypotheses in the preregistration of this study. However, we did not find support for our hypotheses that larger errors in timing are associated with more impulsive subjects in the short delay task, nor did we find a significant increase in correlation between short and long delay discount factors after accounting for subjective timing.

We found that accounting for ICS $e$ only explained additional variance in discounting in the new group but not the follow-up group, which highlights the importance of temporal proximity of the timing and the intertemporal tasks. One possible explanation for this difference is that, for the new group, the discounting task influenced performance on the subsequent timing task. Indeed, Panfil, Bailey, Davis, Mains, and Kirkpatrick (2020) showed that if a timing task precedes an intertemporal choice task it can decrease impulsive choices. However, since the distribution of ICS in the new and follow-up groups were highly overlapping, this explanation is unsupported by the data. Another possible explanation for this difference is that both ICS and discounting are somewhat unstable over time. For example, both timing and discounting can be perturbed by emotional statuses (Lin \& Epstein, 2014; Paasche, Weibel, Wittmann, \& Lalanne, 2019) or caloric intake (Fung, Murawski, \& Bode, 2017; Wang \& Huangfu, 2017). These and other factors could contribute to moderate test-retest reliability for discounting (Beck \& Triplett, 2009) or timing (Bégel, Verga, Benoit, Kotz, \& Dalla Bella, 2018) which would wash out the small effects observed here.

We used three different measures of internal clock speed, without a strong a priori belief about which would influence choices in the delay discounting task: time perception (ICS e), time production (ICS p), ICS errors (ICS Err). We found only time perception $(I C S e)$ to be associated with discounting in the seconds task. Sensory timing (duration discrimination, perception) and motor timing (production, reproduction of the time interval) putatively have distinct underlying neural mechanisms (Paton \& Buonomano, 2018). We note that in both the time perception and the intertemporal choice tasks time is controlled by the experimenter which makes these two experiments closer to each other, in comparison to the production task where timing is controlled by the subject. Moreover, time estimation is intertwined in the short delay task: you may experience the delay in seconds once, but then decide to wait or not on another similar trial (update your belief) based on your subjective experience of the elapsed time. In the time production experiment, time is controlled (started and ended) by the subject. As van den Broek et al. (1992) puts it, production tasks involve not only temporal judgement but also an ability to withhold a response: taking an action to indicate the end of the interval. Therefore, time production performance may not be indicative of "deficient temporal discrimination per se, since their performance 
might be attributable to difficulty in inhibiting responding" (Mischel \& Underwood, 1974).

Our preregistered definition of ICS Err combines both perception and production task measurements. We did not find any correlation of the distortions in timing as a whole to the discount factors, nor did we find any significant correlation when ICS error was calculated using just the time perception task in the sample of college students. We speculate that a clinical sample with high levels of impulsivity might be a better one to relate to a less accurate ICS (higher ICSErr) as previous works suggest: to an impulsive person the passage of time may appear to be more intolerable and more error prone (Paton \& Buonomano, 2018). For example, research on children (in the context of attentiondeficit/hyperactivity disorder or preterm birth) suggests that there is a positive relationship between waiting time in the delay of gratification task and children's performance in the timing task (Hüning et al., 2017; Reinelt, Wirth, Rauch, \& Gawrilow, 2014; Sonuga-Barke, Bitsakou, \& Thompson, 2010; Zmyj, 2018).

In contrast to previous published work, we did not find a strong relationship between timing and intertemporal choice. It is worth noting that the previous studies investigating the relationship between these two phenomena sometimes found positive (Baumann \& Odum, 2012) and sometimes found negative (Corvi et al., 2012; Sharma \& Khan, 2018) correlations between internal clock speed and discount factors. Considering our preregistered findings along with the inconsistency in the direction of the relationship in previously published studies, one possible conclusion is that there is no reliable relationship between individual differences in timing and intertemporal choice, despite the intuitive appeal of such a relationship. To definitively resolve this question, a larger preregistered multi-site study (similar to Hagger et al., 2016) should be done including both neurotypical and clinical populations, where more extreme examples of individual differences in timing and intertemporal choice might reveal a connection.

\section{Author Contributions}

All authors contributed to the study design, interpretation of the results and writing the manuscript. Data collection and analyses were performed by E. Lukinova under the supervision of J. Erlich.

\section{References}

Bates, D., Maechler, M., Bolker, B., \& Walker, S. (2014). lme4: Linear mixed-effects models using eigen and $\mathrm{s} 4$. $R$ package version, 1(7), 1-23.

Baumann, A. A., \& Odum, A. L. (2012, July). Impulsivity, risk taking, and timing. Behavioural Processes, 90(3), 408-414. doi: 10.1016/j.beproc.2012.04.005
Beck, R. C., \& Triplett, M. F. (2009). Testretest reliability of a group-administered paperpencil measure of delay discounting. Experimental and Clinical Psychopharmacology, 17(5), 345-355. doi: 10.1037/a0017078

Bégel, V., Verga, L., Benoit, C.-E., Kotz, S. A., \& Dalla Bella, S. (2018, November). Test-retest reliability of the Battery for the Assessment of Auditory Sensorimotor and Timing Abilities (BAASTA). Annals of Physical and Rehabilitation Medicine, 61(6), 395-400. doi: 10.1016/j.rehab.2018 .04 .001

Bradford, W. D., Dolan, P., \& Galizzi, M. M. (2019, February). Looking ahead: Subjective time perception and individual discounting. Journal of Risk and Uncertainty, 58(1), 43-69. doi: 10.1007/s11166-019-09298-1

Bürkner, P.-C. (2017). brms: An R Package for Bayesian Multilevel Models Using Stan. Journal of Statistical Software, 80(1), 1-28. doi: 10.18637/jss.v080.i01

Carpenter, B., Gelman, A., Hoffman, M., Lee, D., Goodrich, B., Betancourt, M., ... Riddell, A. (2016). Stan: A probabilistic programming language. Journal of Statistical Software, 20, 1-37.

Church, R. M. (1984). Properties of the internal clock a. Annals of the New York Academy of Sciences, 423(1), 566-582.

Cohen, J. (2013). Statistical power analysis for the behavioral sciences. Academic press.

Corvi, A. P., Juergensen, J., Weaver, J. S., \& Demaree, H. A. (2012, December). Subjective time perception and behavioral activation system strength predict delay of gratification ability. Motivation and Emotion, 36(4), 483-490. doi: 10.1007/s11031-011-9275-0

Diedenhofen, B., \& Musch, J. (2015). cocor: A comprehensive solution for the statistical comparison of correlations. PloS one, 10(4), e0121945.

Fung, B. J., Murawski, C., \& Bode, S. (2017, November). Caloric primary rewards systematically alter time perception. Journal of Experimental Psychology: Human Perception and Performance, 43(11), 1925-1936. doi: 10.1037/ xhp0000418

Gabry, J. (2015). Shinystan: interactive visual and numerical diagnostics and posterior analysis for bayesian models. $R$ Package Version, 2(0).

Glicksohn, J., \& Hadad, Y. (2012, January). Sex Differences in Time Production Revisited. Journal of Individual Differences, 33(1), 35-42. doi: 10.1027/1614-0001/a000059

Guo, J., Lee, D., Sakrejda, K., Gabry, J., Goodrich, B., De Guzman, J., ... Fletcher, J. (2016). rstan: $\mathrm{R}$ interface to stan. $R, 534$, $0-3$.

Hagger, M. S., Chatzisarantis, N. L. D., Alberts, H., Anggono, C. O., Batailler, C., Birt, A. R., ... Zwienenberg, M. (2016, July). A Multilab Preregistered Replication of the EgoDepletion Effect. Perspectives on Psychological Science, 11(4), 546-573. doi: 10.1177/1745691616652873

Hlavac, M. (2018). stargazer: well-formatted regression and summary statistics tablesr package version 5.2. $1 \mathrm{https}: / / \mathrm{cran} . R$ project. org/package $=$ stargazer.

Huang, L., Rattner, A., Liu, H., \& Nathans, J. (2013, March). How to draw the line in biomedical research. eLife, 2. doi: 10.7554/eLife.00638 
Hüning, B., Assing, B., Weishaupt, E., Dransfeld, F., FelderhoffMüser, U., \& Zmyj, N. (2017, December). Delay of gratification and time comprehension is impaired in very preterm children at the age of 4 years. Early Human Development, 115, 77-81. doi: 10.1016/j.earlhumdev.2017.09.018

Karmarkar, U. R., \& Buonomano, D. V. (2007, February). Timing in the Absence of Clocks: Encoding Time in Neural Network States. Neuron, 53(3), 427-438. doi: 10.1016/ j.neuron.2007.01.006

Kim, B. K., \& Zauberman, G. (2009). Perception of anticipatory time in temporal discounting. Journal of Neuroscience, Psychology, and Economics, 2(2), 91-101. doi: 10.1037/a0017686

Lin, H., \& Epstein, L. H. (2014). Living in the moment: Effects of time perspective and emotional valence of episodic thinking on delay discounting. Behavioral Neuroscience, 128(1), 1219. doi: $10.1037 / \mathrm{a} 0035705$

Lucci, C. R. (2013). Time, Self, and Intertemporal Choice. Frontiers in Neuroscience, 7. doi: 10.3389/fnins.2013.00040

Lukinova, E., Wang, Y., Lehrer, S. F., \& Erlich, J. C. (2019, February). Time preferences are reliable across time- horizons and verbal versus experiential tasks. eLife, 27. doi: https://doi.org/10.7554/eLife.39656.001

Matthews, W. J. (2011, May). Stimulus Repetition and the Perception of Time: The Effects of Prior Exposure on Temporal Discrimination, Judgment, and Production. PLoS ONE, 6(5), e19815. doi: 10.1371/journal.pone.0019815

Mioni, G., Stablum, F., McClintock, S. M., \& Grondin, S. (2014, April). Different methods for reproducing time, different results. Attention, Perception, $\mathcal{F}$ Psychophysics, 76(3), 675681. doi: 10.3758/s13414-014-0625-3

Mischel, W., \& Underwood, B. (1974). Instrumental ideation in delay of gratification. Child development, 1083-1088.

Paasche, C., Weibel, S., Wittmann, M., \& Lalanne, L. (2019, November). Time perception and impulsivity: A proposed relationship in addictive disorders. Neuroscience $\mathcal{E}$ Biobehavioral Reviews, 106, 182-201. doi: 10.1016/j.neubiorev .2018.12.006

Panfil, K., Bailey, C., Davis, I., Mains, A., \& Kirkpatrick, K. (2020, February). A time-based intervention to treat impulsivity in male and female rats. Behavioural Brain Research, 379, 112316. doi: 10.1016/j.bbr.2019.112316

Paton, J. J., \& Buonomano, D. V. (2018, May). The Neural Basis of Timing: Distributed Mechanisms for Diverse Functions. Neuron, 98(4), 687-705. doi: 10.1016/j.neuron.2018 .03 .045

Peirce, J. W. (2007). PsychoPypsychophysics software in Python. Journal of neuroscience methods, 162(1), 8-13.

Rattat, A.-C., \& Droit-Volet, S. (2012, March). What is the best and easiest method of preventing counting in different temporal tasks? Behavior Research Methods, 44(1), 67-80. doi: 10.3758/s13428-011-0135-3

Ray, D., \& Bossaerts, P. (2011). Positive Temporal Dependence of the Biological Clock Implies Hyperbolic Discounting. Fron- tiers in Neuroscience, 5. doi: 10.3389/fnins.2011.00002

Reinelt, T., Wirth, A., Rauch, W., \& Gawrilow, C. (2014, March). Duration Discrimination Predicts Delay of Gratification In Children with and without ADHD. Procedia - Social and Behavioral Sciences, 126, 220-221. doi: 10.1016/j.sbspro .2014 .02 .383

Rung, J. M., Frye, C. C., DeHart, W. B., \& Odum, A. L. (2019, November). Evaluating the effect of delay spacing on delay discounting: carry-over effects on steepness and the form of the discounting function. Journal of the experimental analysis of behavior, 112(3), 254-272. doi: 10.1002/jeab.556

Schultz, W. (2010, May). Subjective neuronal coding of reward: temporal value discounting and risk: Subjective coding of reward value and risk. European Journal of Neuroscience, 31(12), 2124-2135. doi: 10.1111/j.1460-9568.2010.07282 . $\mathrm{X}$

Sharma, S. N., \& Khan, A. (2018, November). Interval timing predicts impulsivity in intertemporal choice: combined behavioral and drift-diffusion model evidence. Journal of Cognitive Psychology, 30(8), 816-831. doi: 10.1080/20445911 .2018 .1539002

Sonuga-Barke, E., Bitsakou, P., \& Thompson, M. (2010, April). Beyond the Dual Pathway Model: Evidence for the Dissociation of Timing, Inhibitory, and Delay-Related Impairments in Attention-Deficit/Hyperactivity Disorder. Journal of the American Academy of Child $\mathcal{E}$ Adolescent Psychiatry, 49(4), 345-355. doi: 10.1016/j.jaac.2009.12.018

Takahashi, T., Oono, H., \& Radford, M. H. (2008, March). Psychophysics of time perception and intertemporal choice models. Physica A: Statistical Mechanics and its Applications, 387(8-9), 2066-2074. doi: 10.1016/j.physa.2007.11 .047

van den Broek, M., Bradshaw, C., \& Szabadi, E. (1992, February). Performance of impulsive and non-impulsive subjects on two temporal differentiation tasks. Personality and Individual Differences, 13(2), 169-174. doi: 10.1016/0191-8869(92) 90039-R

Wang, X. X.-T., \& Huangfu, G. (2017, February). Glucosespecific signaling effects on delay discounting in intertemporal choice. Physiology E Behavior, 169, 195-201. doi: 10.1016/j.physbeh.2016.12.001

Wittmann, M., \& Paulus, M. P. (2008, January). Decision making, impulsivity and time perception. Trends in Cognitive Sciences, 12(1), 7-12. doi: 10.1016/j.tics.2007.10.004

Zauberman, G., Kim, B. K., Malkoc, S. A., \& Bettman, J. R. (2009, August). Discounting Time and Time Discounting: Subjective Time Perception and Intertemporal Preferences. Journal of Marketing Research, 46(4), 543-556. doi: $10.1509 / j m k r .46 .4 .543$

Zmyj, N. (2018, July). The relationship of delay of gratification and time comprehension in 4-year-old children. International Journal of Behavioral Development, 42(4), 434-438. doi: $10.1177 / 0165025417727870$ 